\title{
Combination of the State Equation with thermodynamic equilibrium to improve characterization of variated oil properties
}

\author{
An Hai Nguyen *, Duc Hoang Nguyen \\ PetroVietnam Exploration Production Corporation, Hanoi, Vietnam
}

ARTICLE INFO ABSTRACT

Article history:

Received $18^{\text {th }}$ Feb. 2021

Revised 09th May 2021

Accepted 09st June 2021

\section{Keywords:}

Equation of state,

Fluid simulation,

PVT,

Thermodynamic equilibrium.
For oil and gas fields with complex fluid behavior, the PVT properties of the fluid continuously change spatially in the reservoir (both in spacial and depth). While using common method to determine the composition variation and to build the fluid model more accurately under reservoir conditions, it is necessary to divide into sub-regions, and to collect and analyze numerous fluid samples. This paper presents the application Equation of state in thermodynamic equilibrium has provided an effective method for modeling the fluid properties of such oil and gas reservoir. In this way, it is possible to model the fluid properties for each specific location in the space (continuous change) to minimize the need to carry out the sampling and analysis of additional fluid samples.

${ }^{*}$ Corresponding author 


\title{
Tạp chí Khoa học Kỹ thuật Mỏ - Địa chất
}

Trang điện tử: http://tapchi.humg.edu.vn

\section{Kết hợp phương trình trạng thái với cân bằng nhiệt động học cho việc mô phỏng chính xác phân bố đặc tính chất lưu trong vỉa chứa dầu khí có động thái lưu biến phức tạp}

\author{
Nguyễn Hải An*, Nguyễn Hoàng Đức \\ Tổng công ty Thăm dò Khai thác Dầu khí, Hà Nội, Việt Nam
}

THÔNG TIN BÀI BÁO

Quá trình:

Nhận bài 18/02/2021

Sửa xong 09/5/2021

Chap nhận đăng 09/6/2021

\section{Từ khóa:}

Cân bằng nhiệt động học,

Mô hình hóa chất lưu,

PVT,

Phương trình trạng thái.

\section{TÓM TẮT}

Đối với các mỏ dầu khí chứa chất lưu với động thái tính chất lưu biến biến đổi phức tạp, tính chất PVT của chất lư liên tục thay đổi theo không gian trong vỉa chứa (cả về diện và chiều sâu). Nếu chỉ sử dụng các phương trình trạng thái (EOS) để mô hình hóa chất lưu theo cách thông thường, cần phải thu thập và phân tích rất nhiều mẫu chất lưu để xác đinh sư biến đổi thành phần chất lưu trong không gian vỉa chứa mà kết quả vẫn không chính xác hoàn toàn. Bài báo trình bày phương pháp úng dụng kết hợp giữa phương trình trạng thái và điều kiên cân bằng nhiêt động học để mô hình hóa đặc tính chất lưu của vỉa chứa dầu khí có động thái lưu biến phức tạp. Phương pháp này cho phép mô hình hóa đặc tính chất lư cho tùng vị trí cụ thể trong không gian (biến đổi liên tuc) sát với thực tếcũng nhu giảm thiểu tối đa việc phải thu thập và phân tích thêm mẫu chất lư nhăm giảm hoặc tiết kiệm chi phí.

C 2021 Trường Đại học Mỏ - Địa chất. Tất cả các quyền được bảo đảm.

\section{Mở đầu}

Tính chất của chất lưu trong vỉa chứa có ảnh hưởng rất lớn đến việc thiết kế và tối ưu các chiến lược khai thác/bơm ép và hệ thống thiết bị bề mặt để quản lý thu hồi dầu khí một cách hiệu quả. Việc mô hình hóa đặc tính các chất lưu không chính xác sẽ dẫn đến các rủi ro và sai số lớn về đánh giá chế độ khai thác, động thái dòng chảy và chiến lược thu hồi dầu, gây ảnh hưởng lớn đến kết quả xác định giá trị thương mại của mỏ.

\footnotetext{
*Tác giả liên hệ

E - mail: annh1@pvep.com

DOI: 10.46326/JMES.2021.62(3a).01
}

Với những vỉa chứa dầu khí bị phân thành nhiều khối trong điều kiện nhiệt động (áp suất và nhiệt độ) khác nhau, tính chất PVT biến đổi phức tạp: GOR (tỷ lệ khí - dầu) khác nhau ở các giếng và khu vực, áp suất bão hòa, độ nhớt thay đổi,... Theo cách mô hình hóa thông thường, sẽ phải chia ra rất nhiều các phân vùng thủy động lực phù hợp với điều kiện nhiệt động. Đồng thời, phải tiến hành thu thập và phân tích thêm rất nhiều mẫu chất lưu (trong mỗi vùng, ít nhất thu thập được thông tin của một giếng khai thác) mà kết quả thu được vẫn chưa hoàn toàn chính xác. Ngoài ra, mức độ chi tiết bị giới hạn bởi số vùng chia hữu hạn, trong khi thực tế xét về mặt không gian thì tính chất PVT của chất lưu trong vỉa chứa của mỏ có sự biến đổi liên tục. Để giải quyết vấn đề này, nhóm tác giả tiến hành 
nghiên cứu kết hợp giữa phương trình trạng thái và điều kiện cân bằng nhiệt động học để mô hình hóa đặc tính chất lưu của vỉa chứa dầu khí có động thái lưu biến phức tạp.

\section{Phương trình trạng thái và cân bằng nhiệt động học}

\subsection{Phương trình trạng thái}

Bất cứ phương trình nào thể hiện mối liên quan giữa trạng thái pha của hỗn hợp (hoặc chất) với áp suất, nhiệt độ và thể tích đều được gọi là phương trình trạng thái (EOS) (Ahmed, 2007). Bắt đầu từ định luật Boyle - Charles sử dụng cho khí lý tưởng biểu thị bằng công thức sau:

$$
P V=n R T
$$

Trong đó: $P$ - áp suất (psia); $V$ - thể tích ( $\left.\mathrm{ft}^{3}\right) ; n$ - số mol khí ở điều kiện áp suất $P$, nhiệt độ $T$ có thể tích $V$ (mol); $T$ - nhiệt độ $\left({ }^{0} \mathrm{R}\right) ; R$ - hằng số khí, $R=10,732$ (psia.scf/lb. mol. ${ }^{\mathrm{R}} \mathrm{R}$ ).

Bảng 1. Các phương trình trạng thái EOS phổ biến trong công nghiệp dầu khí.

\begin{tabular}{|c|c|}
\hline $\begin{array}{c}\text { Peng - Robinson } \\
\text { PR-1976 }\end{array}$ & $\frac{a(T) V}{R T[V(V+b)+b(V-b)}$ \\
\hline Fuller-1976 & $\frac{a(T)}{R T(V+c b)}$ \\
\hline $\begin{array}{c}\text { Heyen-1980 } \\
\text { Sandler-1994 }\end{array}$ & $\frac{a(T) V}{\left.R T\left[V^{2}+b(T)+c\right) V-b(T) c\right]}$ \\
\hline $\begin{array}{c}\text { Schmit \& Wenzel } \\
\text {-1980 }\end{array}$ & $\frac{a(T) V}{R T\left(V^{2}+u b V+w b^{2}\right)}$ \\
\hline $\begin{array}{c}\text { Kubic-1982 } \\
\text { Adachi-1983 }\end{array}$ & $\frac{a(T) V}{R T(V+c)^{2}}$ \\
\hline $\begin{array}{c}\text { Stryjeck \& Vera } \\
\text { SV-1986 }\end{array}$ & $\frac{a(T) V}{\left.R T\left(b^{2}+2 b V-b^{2}\right)\left(V+b_{3}\right)\right]}$ \\
\hline $\begin{array}{c}\text { Schwartzentrube } \\
\text { r \& Renon -1989 }\end{array}$ & $\frac{a(T)}{R T[(V+c)(V+2 c+b)]}$ \\
\hline
\end{tabular}

Trong đó: $a, b, c$ - hệ số tương quan được tính toán theo áp suất tới hạn và nhiệt độ tới hạn; $w$ - hệ số lệch cầu.

Ban đầu, phương trình (1) chỉ được áp dụng chủ yếu cho các cấu tử tinh khiết, sau đó được phát triển cho các hợp chất nhiều thành phần hơn. Khi mới áp dụng cho hỗn hợp, chúng được sử dụng cho các hỗn hợp không phân cực (các EOS: Soave-1972; Peng \& Robinson-1976) và các hợp chất phân cực yếu (các EOS: Huron-1978; Assenlineau-1978; Graboski \& Daubert-1978) (tác giả Ahmed tổng quan lại năm 2007 trong sách tham khảo chuyên ngành công nghệ mỏ dầu khí). Theo thời gian, phương trình trạng thái đã phát triển nhanh chóng cho việc tính toán đặc tính chất lưu PVT trong công nghiệp dầu khí của các hỗn hợp cả phân cực lẫn không phân cực.

Có nhiều điểm thuận tiện cho việc áp dụng phương trình trạng thái trong việc tính toán đặc tính chất lưu: i) dùng được với khoảng áp suất và nhiệt độ rộng, kể cả vùng áp suất cao; ii) các cấu tử có thể thay đổi bất kỳ, từ nhẹ tới nặng, từ không phân cực tới phân cực; iii) có thể dùng cho cân bằng lỏng - hơi, lỏng - lỏng; iv) có thể dùng cho cả pha hơi lẫn pha lỏng; Ngoài ra, các phương trình trạng thái còn dùng để xác định các tính chất như tỷ trọng, áp suất hoi, entanpy, entropy,...

Phương trình Van Der Waals là phương trình trạng thái đầu tiên dự đoán sự cùng tồn tại hai pha lỏng - hơi. Sau đó, lần lượt các EOS như Redlich \& Kwong-1949, Soave-1972, Peng \& Robinson-1976, Carhanan và Starling-1969, Guggenheim -1965, Boublik-1981, Chen \& Kreglewski-1977, Christoforakos và Franck-1986, Heilig và Franck 1989 (tác giả Ahmed tổng quan lại năm 2007 trong sách tham khảo chuyên ngành công nghệ mỏ dầu khí) cải tiến để tăng độ chính xác của phương trình trạng thái trong việc dự đoán cân bằng pha, tính áp suất hơi, tỷ trọng lỏng, tỷ trọng khí, tỷ lệ các pha cân bằng,... trong công nghiệp khai thác dầu khí (Bảng 1).

Đối với hệ nhiều cấu tử, các tham số $\mathrm{a}, \mathrm{b}, \mathrm{c}, \mathrm{w}$ của hỗn hợp được xác định dựa trên tính chất và hàm lượng thành phần của từng cấu tử theo công thức sau:

$$
\begin{aligned}
& a_{h h}=\sum_{i=1}^{n} n_{i} a_{i} ; b_{h h}=\sum_{i=1}^{n} n_{i} b_{i} \\
& c_{h h}=\sum_{i=1}^{n} n_{i} c_{i} ; w_{h h}=\sum_{i=1}^{n} n_{i} w_{i}
\end{aligned}
$$

Trong đó: $a_{i}, b_{i} c_{i}$ - hệ số tương quan được tính theo áp suất tới hạn và nhiệt độ tới hạn của cấu tử $I ; w_{i}$ - hệ số lệch cầu của cấu tử $i ; n_{i}$ - hàm lượng mol cấu tử $i$. 
Như vậy, nếu như xác định được hàm lượng thành phần của từng cấu tử, bằng phương trình trạng thái sẽ mô phỏng và dự báo được đặc tính lưu biến của hỗn hợp theo điều kiện áp suất - thể tích -nhiệt độ (PVT).

\subsection{Cân bằng nhiệt động học}

Cân bằng nhiệt động giữa lỏng và hơi của cấu tử $i$ được biểu thị như sau (Whitson và Brule, 2000):

$$
f_{i}^{V}=f_{i}^{L}
$$

Trong đó: $\mathrm{f}_{i}^{V}, \mathrm{f}_{i}^{L}$ - độ bay hơi của cấu tử $i$ trong pha hơi và pha lỏng tương ứng (psi) .

Tuy nhiên, trong thực tế, thường sử dụng phương trình sau:

$$
K_{i}=\frac{y_{i}}{x_{i}}
$$

Trong đó: $K_{i}$ - hằng số cân bằng pha lỏng - hơi của cấu tử $\mathrm{i}$, bằng tỷ lệ giữa phần mol của cấu tử $i$ trong pha hơi $y_{i}$ và phần mol của nó trong pha lỏng - $x_{i}$. Phương trình này còn được gọi là định luật Henry và hằng số $K$ được biểu thị giống như hằng số Henry.

Phụ thuộc vào từng hệ thống mà có thể có một số phương pháp xác định hằng số $\mathrm{K}$ được sử dụng. Thực tế cho thấy, phương pháp đo thực nghiệm được ưa chuộng nhất (Benedict và nnk., 1940), nhưng phương pháp này tương đối tốn kém và mất thời gian. Ngoài ra, một vài công cụ đồ thị (graphical tool) và phương pháp số (numerical tool) cũng được dùng để xác định hằng số $K$.

Thông thường, giá trị của hằng số K là một hàm của áp suất, nhiệt độ và thành phần pha lỏng - hơi. Tuỳ theo các cấu tử có trong hệ thống, áp suất, nhiệt độ, thành phần và mức độ phân cực hoá mà có thể dùng các phương pháp khác nhau. Các phương pháp được dùng rộng rãi là giản đồ $K$, định luật Raoult, áp dụng phương trình trạng thái. Dựa vào phương trình cân bằng nhiệt động học, từ thành phần hô̂n hợp ban đầu, thành phần các cấu tử trong hỗn hợp sẽ được tính toán tùy thuộc vào điều kiện nhiệt độ, áp suất bất kỳ (Hanafy và Mahgoub, 2005).

Trong tính toán cân bằng lỏng - hơi của dòng hai pha, chỉ có số liệu áp suất, nhiệt độ và thành phần chung của hỗn hợp. Kết quả cần tính bao gồm: i) tỷ lệ lỏng / hơi; ii) thành phần các cấu tử trong pha lỏng và thành phần các cấu tử trong pha hoi.

Do thành phần của 2 pha lỏng và hơi chưa biết nên để khởi tạo quy trình tính toán cần giả sử thành phần các pha ban đầu như sau:

- Với pha lỏng: cấu tử nặng nhất =0,9999 $\%$ mol; các cấu tử còn lại $=0,0001 \%$ mol;

- Vói pha khí: cấu tử nhẹ nhất = 0,9999%mol; các cấu tử còn lại $=0,0001 \%$ mol.

Ngoài ra, tỷ lệ lỏng / dòng hai pha ban đầu cũng cần được giả định. Tỷ lệ này sẽ được tính theo phương pháp chia đôi lấy giá trị trung bình và hiệu chỉnh dần qua từng vòng lặp. Sau khi xác định $\mathrm{K}_{\mathrm{i}}$, thành phần pha lỏng, pha hơi tương ứng sẽ được xác định bằng các phương trình (3), (4) và hiệu chỉnh dần qua vòng lặp ngoài cho đến khi đạt giá trị sai số $(\varepsilon)$ theo yêu cầu. Cụ thể sơ đồ thuật toán như Hình 1.

$G_{o}$ và $G_{t b}$ được tính theo công thức (5) dưới đây tương ứng với $L_{o}$ và $L_{t b}(V=1-L)$ :

$$
\begin{gathered}
G=\sum y_{i}-\sum x_{i} \\
=\sum z_{i} \frac{K_{i}-1}{V\left(K_{i}-1\right)+1}=0
\end{gathered}
$$

Với: $G$ - biến động năng lượng tự do Gibb ( $\mathrm{Kj} / \mathrm{mol}$ ), tại trạng thái cân bằng pha lỏng-hơi thì $G$ $=0$.

\section{3. Ứng dụng chính xác hóa phân bố đặc tính dầu khí, đối tượng $H$ thuộc vùng Touggourt, sa mạc Sahara}

Vỉa dầu $H$ nằm trong vùng Touggourt (Algeria), thuộc sa mạc Sahara, cách thủ đô Alger khoảng 500 km về phía đông nam. Cập nhật động thái khai thác và phân tích PVT các mẫu ở những giếng mới khoan có thể thấy vỉa bị phân thành nhiều khối khác nhau, tính chất PVT của mỏ biến đổi phức tạp như GOR khác nhau ở các giếng, khu vực, áp suất bão hòa, độ nhớt thay đổi (Bảng 2).

Các thông tin từ Bảng 2 cho thấy: đối tượng tầng $H$ chứa dầu loại nhẹ với tỷ trọng từ $42 \div 45^{0}$ API, cùng đó là tỷ số khí - dầu biến đổi từ $124 \div 180$ $\mathrm{sm}^{3} / \mathrm{sm}^{3}$. Hệ số thể tích dầu thay đổi từ $1,35 \div 1,69$ $\mathrm{rm}^{3} / \mathrm{sm}^{3}$. Dầu trong điều kiện vỉa ban đầu ở trạng thái chưa bão hòa với áp suất điểm bọt trong khoảng $1608 \div 2500$ psi. Độ nhớt thay đổi từ $0,2 \div 0,5 \mathrm{cp}$. 


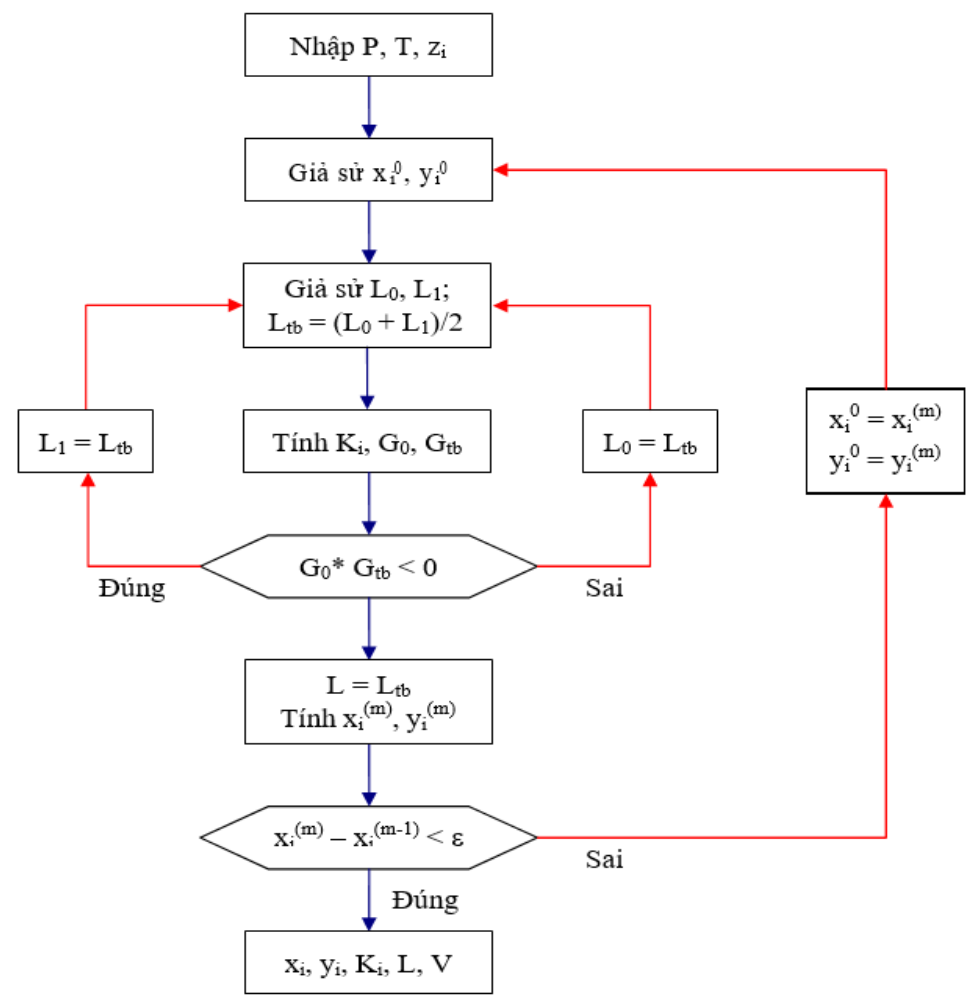

Hình 1. Sơ đồ thuật toán tính hàm lượng các cấu tủ.

Zi: hàm lượng cấu tử thứ i trong hỗn hợp; L: phần pha lỏng; $V=1$ - L; chỉ số '0', '1': giá trị ban đầu và giá trị tính toán tiếp sau; chỉ số 'tb': giá trị trung bình của 2 giá trị trong bước tính toán; chỉ số (m): bước tính toán thứ m.

Bảng 2. Tóm tắt các đặc tính dầu-khí từ các giếng khai thác chất lưu tù̀ vỉa $H$.

\begin{tabular}{|c|c|c|c|c|c|c|c|c|}
\hline TT & Mẫu & $\mathrm{A}$ & $\mathrm{B}$ & $\mathrm{C}$ & $\mathrm{D}$ & $\mathrm{E}$ & $\mathrm{F}$ & $\mathrm{G}$ \\
\hline 1 & Chiều sâu (mTVD) & $\mathrm{x} 630$ & $\mathrm{x} 622$ & $\mathrm{x} 407$ & $\mathrm{x} 460$ & $\mathrm{x} 421$ & $\mathrm{x} 029$ & $\mathrm{x} 683$ \\
\hline 2 & ${\text { Tỷ số khí dầu }\left(\mathrm{sm}^{3} / \mathrm{sm}^{3}\right)}_{174}$ & 126 & 175 & 170 & 178 & 180 & 124 \\
\hline 3 & Áp suất bão hòa $(\mathrm{psi})$ & 2340 & 1608 & 2234 & 2130 & 2123 & 2500 & 2021 \\
\hline 4 & Hệ số thể tích $\left(\mathrm{rm}^{3} / \mathrm{sm}^{3}\right)$ & 1,609 & 1,610 & 1,605 & 1,631 & 1,691 & 1,397 & 1,356 \\
\hline 5 & Mật độ $(\mathrm{API})$ & 42,1 & 43,8 & 42,8 & 42,9 & 42,0 & 44.1 & 44,8 \\
\hline
\end{tabular}

Để giải mô hình hóa đặc tính chất lưu của mỏ, theo cách thông thường thì sẽ phải chia ra nhiều phân vùng thủy lực với đặc trưng PVT riêng. Như vậy, trên toàn diện tích nghiên cứu phải tiến hành thu thập và phân tích thêm rất nhiều mẫu chất lưu (trong mỗi vùng, ít nhất phải có một giếng khai thác thu thập) mà kết quả vẫn chưa hoàn toàn chính xác, chưa kể việc chia nhiều phân vùng sẽ gây ra các lỗi hội tụ trong quá trình chạy mô phỏng khai thác. Đồng thời, mức độ chi tiết bị giới hạn bởi số vùng chia hữu hạn, trong khi thực tế xét về mặt không gian thì đặc tính PVT chất lưu của mỏ có sự biến đổi liên tục.

Bằng cách áp dụng tính toán cân bằng nhiệt động học kết hợp với phương trình trạng thái, bài báo đã đưa ra một phương án hiệu quả cho việc mô hình hóa đặc tính chất lưu cho bất kể vị trí cụ thể trong không gian (biến đổi liên tục) cũng như giảm thiểu tối đa việc thu thập và phân tích thêm mẫu chất lưu (đối với những vùng chưa được lấy mẫu). Nhất là sau thời gian dài khai thác, tính chất và thành phần chất lưu có thể bị biến đổi, dễ gây sai số lớn so với mẫu lấy từ các giếng thăm dò thẩm lượng.

\section{1. Áp dung các quy luật về cân bằng nhiẹt động học để tìm ra phân bố thành phần các cấu tử, tìm xu thế biến đổi các đặc tính PVT tương úng}

Mô hình phương trình trạng thái EOS được xây dựng dựa trên các nguyên lý nhiệt động học cơ bản, do vậy sẽ rất hữu ích cho việc ngoại suy các giá trị vượt ra dải số liệu khảo sát, thí nghiệm. 
Để đảm bảo tính tin cậy, các tham số nhiệt động học trong mô hình PVT được tạo ra dựa trên việc sử dụng các phương trình trạng thái đã được hiệu chỉnh với các số liệu đo từ phòng thí nghiệm. Quá trình mô hình hoá chất lưu theo phương trình trạng thái bao gồm các bước quan trọng sau: tối ưu sốlượng thành phần bằng cách đặc tính hoá các cấu tử $\mathrm{C} 7+$, tính toán cân bằng pha, các phương pháp giải để đảm bảo tính hội tụ, hồi quy với các số liệu thí nghiệm.

Các thành phần nặng C7+ chứa rất nhiều các đồng đẳng khác nhau (parafin, napthen và chất thơm) có ảnh hưởng lớn đến việc xác định động thái pha nhiệt động học của chất lưu. Chẳng hạn như khí condensate, áp suất ngưng tụ bị ảnh hưởng cực kỳ mạnh bởi hàm lượng mol của $\mathrm{C} 7+$. Trong dầu nặng, thành phần $\mathrm{C} 7+$ ảnh hưởng lớn đến độ nhớt, thành tạo asphalten và wax. Tương tự như vậy, trong dầu nhẹ, thể tích dầu và các tính chất khác ở áp suất thấp hơn áp suất bão hòa được xác định bởi hàm lượng các thành phần trung bình và nặng.

Dựa trên số liệu ban đầu, mặc dù đã có đến 9 mẫu chất lưu, nhưng các tham số PVT (thành phần cấu tử $\mathrm{C} 1$ và $\mathrm{C7+}$, GOR, áp suất bão hòa,...) có mối liên hệ kém do chất lượng mẫu và đo chưa đảm bảo, nhiều tạp chất gây bẩn mẫu và ảnh hưởng đến kết quả đo. Trên cơ sở lý thuyết về cân bằng nhiệt động học và phân ly trọng lực, kết hợp với phân tích đánh giá các số liệu thí nghiệm phân tích mẫu PVT từ các giếng khai thác trong vùng nghiên cứu, nhóm tác giả đã loại bỏ được các yếu tố ảnh hưởng, đồng thời tìm ra quy luật về phân bố của những đặc tính PVT quan trọng nhất cũng như phương trình trạng thái EOS để mô phỏng động thái pha (Ahmed, 2007; Jhaveri và Youngren, 1988). Kết quả cho thấy các tính chất quan trọng như hàm lượng thành phần các cấu tử, áp suất bão hòa, tỷ số khí hòa tan,... có quan hệ vói độ sâu vỉa (các Hình 2,3,4). Kết quả mô phỏng phương trình EOS với số liệu phân tích thí nghiệm cho các thông số chính được trình bày ở Hình 5.

\section{2. Áp dụng quy luật phân bố đặc tính lưu thể vào trong mô hình khai thác xác định giá trị PVT}

Trên cơ sở các cấu tử (từ C1 đến C7+; N2, CO2) và các thông số tính chất PVT quan trọng có quan hệ với chiều sâu cùng với phương trình trạng thái "Peng \& Robinson-1976" có độ liên kết tốt nhất (đối với bộ số liệu của vỉa $H$ ), nhóm tác giả đã thực hiện

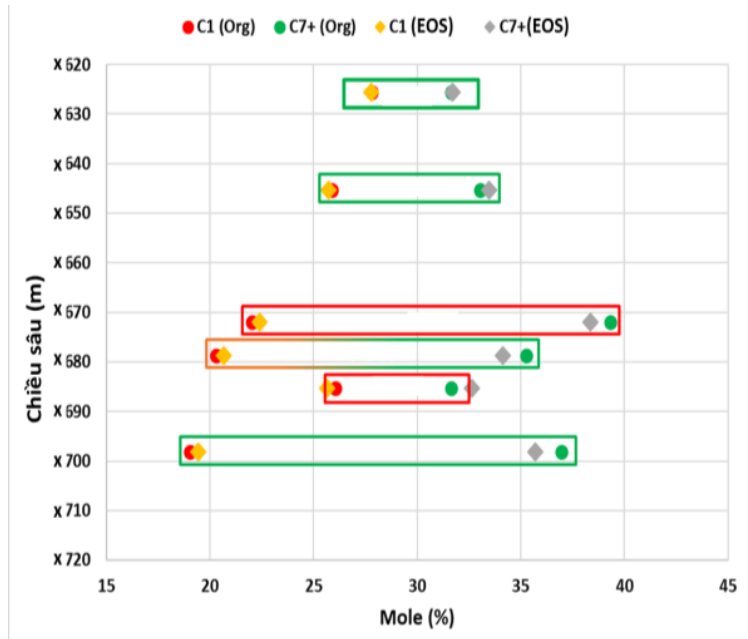

Hình 2. Quan hệ thành phần C1 và C7+ với chiều sâu.

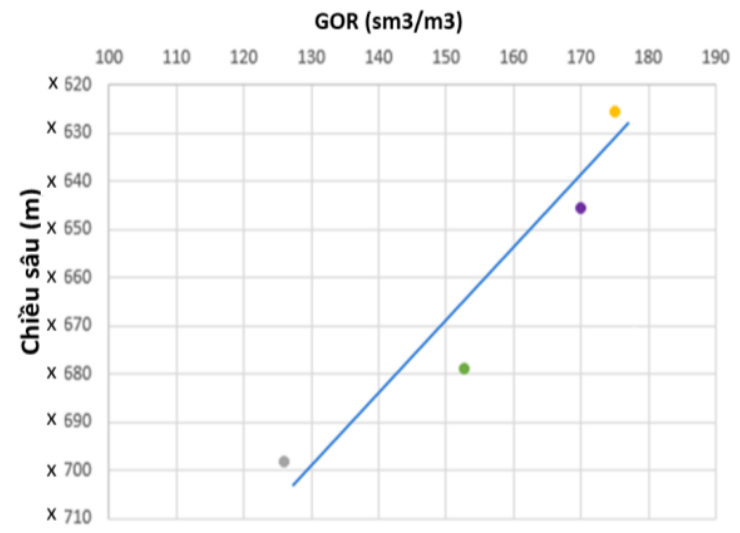

Hình 3. Quan hệ tỷ số khí hòa tan (GOR) với chiều sâu.

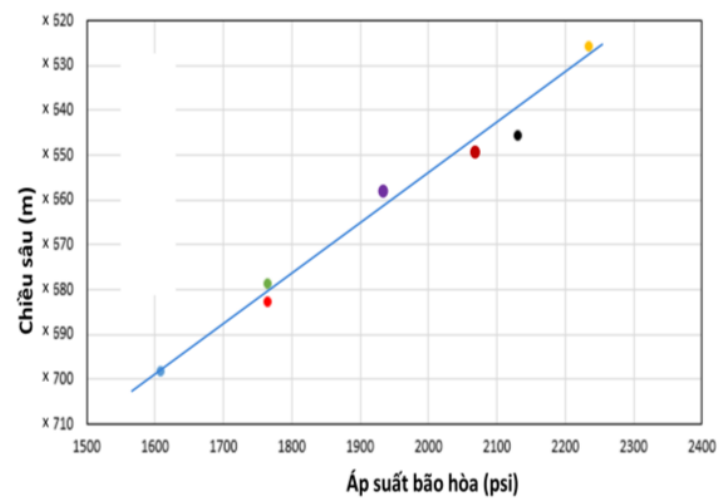

Hình 4. Quan hệ áp suất bão hòa với chiều sâu.

mô hình hóa đặc tính dầu khí điều kiện vỉa bởi hệ thống bảng PVT (Hình 6) để đưa vào sử dụng trong mô hình mô phỏng khai thác vỉa $H$. Trên cơ sở bảng PVT chuẩn theo số liệu thí nghiệm, ứng với mỗi một vị trí cụ thể của mỏ (một ô lưới trong mô hình khai thác), phần mềm mô phỏng sẽ tạo ra một bảng PVT riêng cho ô lưới đó dựa vào chiều sâu. 
Như vậy, bằng việc áp dụng giải pháp EOS đã tạo ra vô số bảng PVT và luôn đảm bảo mỗi ô lưới trong mô hình mô phỏng sẽ có một bảng PVT riêng, không bị hạn chế bởi cách chia số vùng hữu hạn. So sánh với cách áp dụng phương pháp mô hình hóa PVT trước đó (theo cách thông thường, Hình 7), có thể thấy đặc tính PVT của chất lưu, chẳng hạn như áp suất bão hòa, được mô phỏng biến đổi liên tục theo không gian thay vì bị đồng nhất và trung bình hóa cho cả mô hình. Giải pháp này vừa tiết giảm được số lượng mẫu cần thu thập và phân tích, vừa chi tiết hóa được đặc tính PVT của mỏ. Kết quả cũng giúp được việc phục hồi số liệu lịch sử cho tham số GOR cũng như đảm bảo cân bằng vật chất của mô
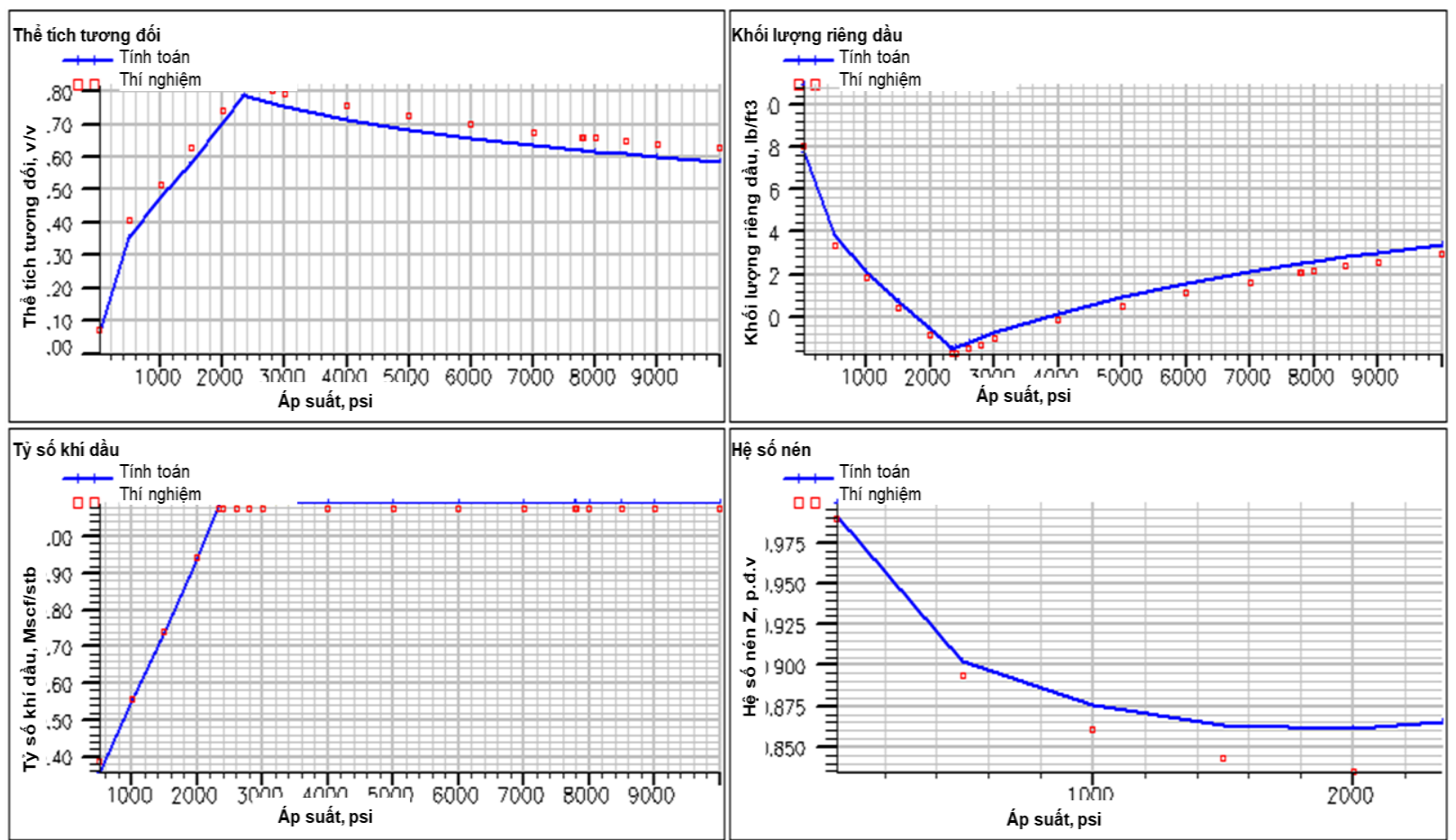

Hình 5. Kết quả khớp số liệu thí nghiệm cho phương trình trạng thái EOS.



Hình 6. Họ bảng PVT của chất lưu vỉa chứa $H$. 
hình khai thác đạt kết quả rất tốt so với cách làm thông thường (Hình 8).

\subsection{Kết luận}

Bằng việc áp dụng nghiên cứu cân bằng nhiệt động học kết hợp với phương trình trạng thái, có thể vừa tiết giảm được số lượng mẫu cần thu thập và phân tích, vừa chi tiết và chính xác hóa được đặc tính PVT của các khu vực quanh các giếng khai thác hiện nay của vỉa chứa $H$ mỏ $B$, đồng thời cũng là cơ sở đế tối ưu việc lấy mẫu và lựa chọn các chỉ tiêu phân tích cho các giếng ở giai đoạn tiếp theo.

Mỗi ô lưới khác nhau sẽ có một bảng PVT riêng (không bị hạn chế bởi cách chia số vùng hữu hạn). Nếu trong mô hình gán mỗi vùng thủy lực 01 bảng PVT thì vẫn cần phải lấy thêm mẫu và phân tích. Tuy nhiên, giá trị của các bảng này sai lệch rất nhiều nên có nguy cơ gây lỗi hội tụ và làm chậm quá trình tính toán. Phương pháp này cũng giúp cho kết quả phục hồi số liệu lịch sử khai thác của mô hình được tốt hơn, đảm bảo hơn. Qua đó, tăng tính tin cậy của mô hình cho công tác dự báo khai thác.
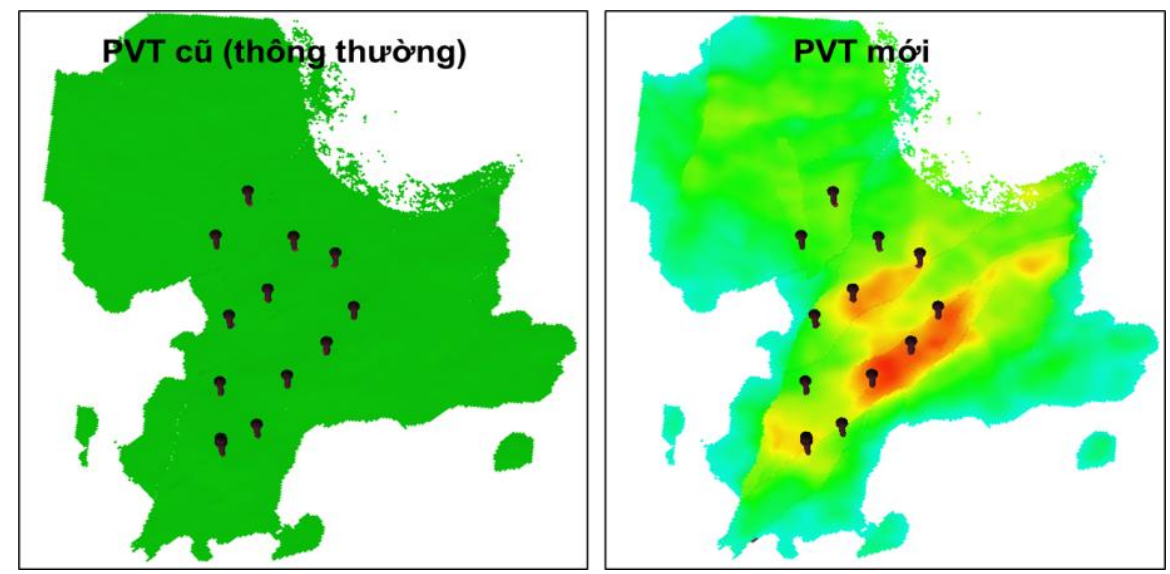

Hình 7. So sánh mô hình hóa đặc tính PVT chất lưu (áp suất bão hòa).

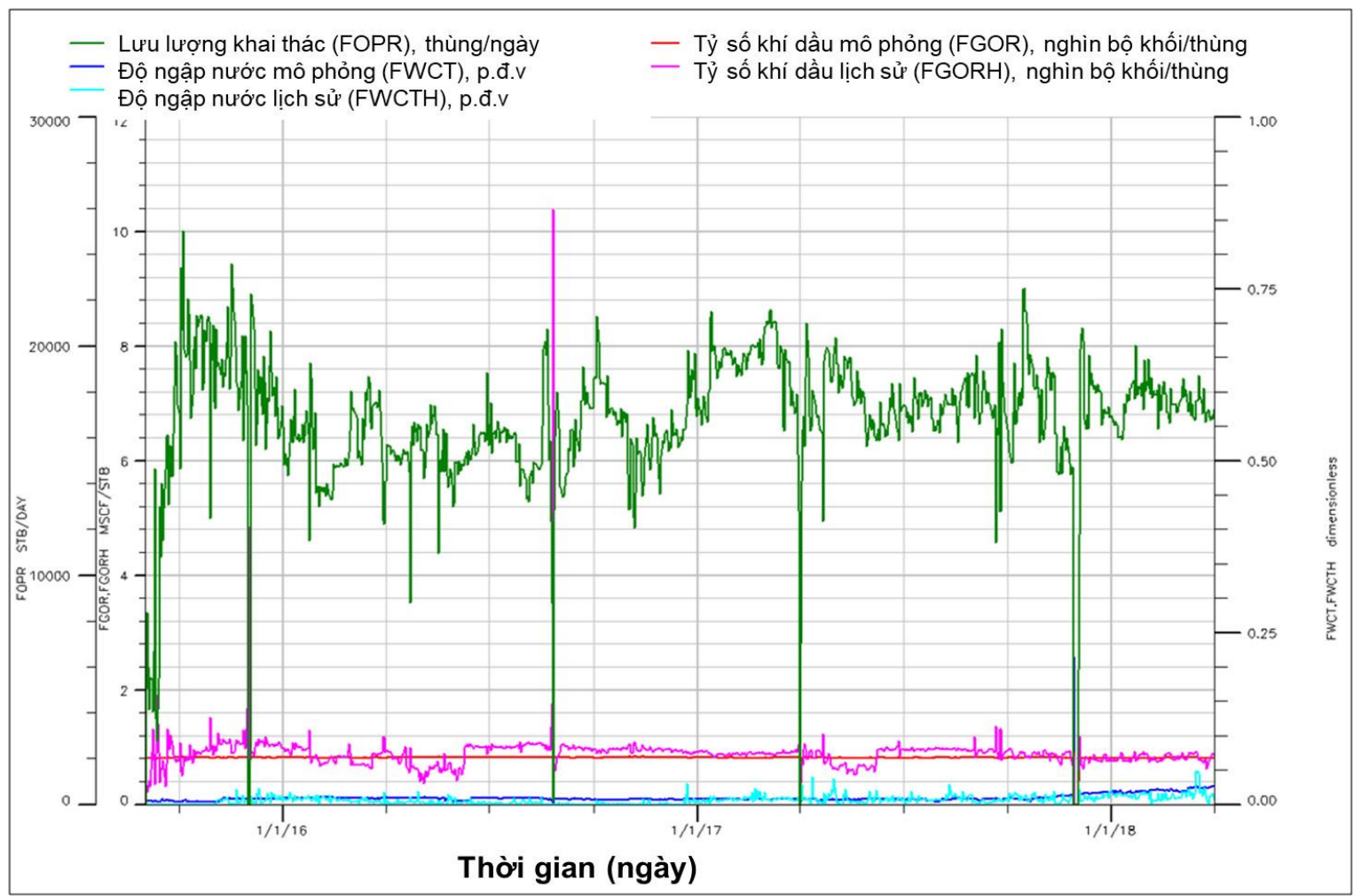

Hình 8. Kết quả phục hồi lịch sử khai thác mô hình vỉa chứa H mỏ B. 


\section{Đóng góp của các tác giả}

Tác giả Nguyễn Hải An xây dựng đề cương bài báo, lên kế hoạch, xử lý số liệu, minh giải và kiểm tra tiến độ công việc. Nguyễn Hoàng Đức thu thập số liệu, phân tích và chỉnh sửa nội dung.

\section{Tài liệu tham khảo}

Ahmed, T. (2007). Equation of state and PVT analysis. Gulf Pbulishing Company. Houston, 553 pages.

Benedict M., Webb G. B. and Rubin L. C. (1940). An empirical equation for thermodynamic properties oflight hydrocarbons and their mixtures: I. Methane, ethane, propane and nbutane, Journal of Chemical Physics, 334 - 345.

Hanafy, H. H. and Mahgoub, I. S. (2005). Methodology of Investgatin the Commpositional Gradient Within the Hydrocarbon Column. Paper SPE 95760, 60 - 65.

Jhaveri B. S. and Youngren G. K. (1988). ThreeParameter Modification of the Peng-Robinson Equation of State To Improve Volumetric Predictions. Society of Petroleum Engineers. 185 - 200.

Whitson, C. H. and Brule, M. R. (2000). Phase Behavior. Monograph Series, SPE. Richardson, Texas 20, 233 pages. 